\title{
A Practical Cryopreservation and Staining Protocol for Immunophenotyping in Population Studies
}

\author{
Helene Barcelo, ${ }^{1}$ Jessica Faul, ${ }^{2}$ Eileen Crimmins, ${ }^{3}$ and Bharat Thyagarajan ${ }^{1}$ \\ ${ }^{1}$ Department of Laboratory Medicine and Pathology, University of Minnesota, Minneapolis, \\ Minnesota \\ ${ }^{2}$ Institute for Social Research Survey Research Center, University of Michigan, Ann Arbor, \\ Michigan \\ ${ }^{3}$ Davis School of Gerontology, University of Southern California Davis, Los Angeles, \\ California
}

Large population-based cohort studies, through their prospective collection of a broad range of health information, represent an invaluable resource for novel insights into the pathogenesis of human diseases. Collection and cryopreservation of viable cells from blood samples is becoming increasingly common in large cohorts as these cells are a valuable resource for immunophenotyping and functional studies. The cryopreservation of peripheral blood mononuclear cells (PBMCs), thawing, and immunophenotyping protocols used to immunophenotype 9938 participants in the Health and Retirement Study (HRS) are described. The extensive quality control involved in a large-scale immunophenotyping epidemiological study is also outlined. The existing literature on the effect of cryopreservation on various immune cell subsets including T, B, NK cells, monocytes, and dendritic cells is provided. (C) 2018 by John Wiley \& Sons, Inc.

Keywords: cryopreservation • flow cytometry • thawing • immunophenotyping • peripheral blood mononuclear cells (PBMCs) • quality control

\section{How to cite this article:}

Barcelo, H., Faul, J., Crimmins, E., \& Thyagarajan, B. (2018). A practical cryopreservation and staining protocol for immunophenotyping in population studies. Current Protocols in Cytometry, 84, e35. doi: 10.1002/cpcy.35

\section{INTRODUCTION}

Large population-based cohort studies prospectively collect a broad range of health information along with social, behavioral, and psychological information on several thousand participants over extended time periods. They represent an invaluable resource for novel insights into the pathogenesis of human diseases and it is estimated that $\sim 1.5$ million volunteers in the U.S.A. have participated in various cohorts funded by the National Institutes of Health (https://www.genome.gov/pages/about/od/ reportspublications/potentialuscohort.pdf). The majority of these studies have successfully collected and stored a variety of biospecimens using standardized protocols to enable measurement of biomarkers to further the understanding of disease etiology, early detection, or prognosis of various diseases. Since appropriate cryopreservation techniques to process and store viable cells are both costly and labor intensive, viable peripheral blood mononuclear cells (PBMCs) are not commonly stored in large population studies. However, PBMCs can be used to study differences in cellular distribution and perform 
functional assays that help understand biological consequences of genetic variants associated with disease outcomes in large-scale genomics studies, several population studies such as the Prostate, Lung, Colorectal and Ovarian (PLCO) Cancer Screening Trial (Landgren et al., 2009), Cancer Prevention Study-3 (CPS-3) (Stevens et al., 2007), Coronary Artery Risk Development in Young Adults (CARDIA) (Friedman et al., 1988; Hughes et al., 1987), large biobanks such as the UK Biobank (500,000 participants) (Elliott, Peakman, \& UK Biobank, 2008), and the All Of Us Research Program (https://allofus.nih.gov/) (1,000,000 participants) have cryopreserved cells for use in future immunophenotyping and functional studies. These studies have used a variety of methods to cryopreserve PBMCs. A majority of studies including PLCO, CPS-3, UK Biobank, and the All Of Us Research program are cryopreserving whole blood with dimethyl sulfoxide (DMSO), while studies such as CARDIA have opted to isolate PBMCs prior to cryopreservation. Several studies have reported that differences in cryopreservation protocols, time intervals between sample collection and processing, and procedures for thawing samples all influence the distribution of cells after cryopreservation (Kutscher et al., 2013; Lemieux, Jobin, Simard, \& Néron, 2017).

Recently, blood samples were collected and processed to obtain cryopreserved PBMCs in the Health and Retirement Study (HRS), a nationally representative longitudinal survey of $>37,000$ individuals over the age of 50 in 23,000 households in all 48 contiguous states in the U.S.A. A multiparameter flow cytometry on cryopreserved PBMCs was performed to estimate 33 immune cell subsets, including T cells, B cells, natural killer (NK) cells, dendritic cells (DC), and monocytes along with $3 \mathrm{~B}$ cell subsets, 19 T cell subsets, $2 \mathrm{NK}$ subsets, 2 DC subsets, and 2 monocyte subsets using two flow cytometers, an LSRII and a FORTESSA X20 (BD Biosciences). Venous blood samples were collected from 9938 HRS participants in $~ 7300$ households during 2016 and 2017. Blood samples were collected directly into a $\mathrm{CPT}^{\mathrm{TM}}$ vacutainer tube (BD Biosciences) at participant homes in all 48 contiguous states in the U.S.A. and shipped daily at room temperature to the Advanced Research and Diagnostics Laboratory (ARDL) at the University of Minnesota. More than $95 \%$ of the samples were processed within $48 \mathrm{hr}$ of sample collection. PBMCs were isolated from venous using the $\mathrm{CPT}^{\mathrm{TM}}$ vacutainer and subsequently cryopreserved and stored for future studies. T cryopreservation, thawing, and immunophenotyping protocols used in the HRS are described.

BASIC PROTOCOL 1

Cryopreservation and Immunophenotyping for Population Studies 2 of 16

\section{CRYOPRESERVATION OF PBMCS USING CPT ${ }^{\mathrm{TM}}$ VACUTAINER AS COLLECTION TUBE}

Isolation of PBMCs from whole blood was performed prior to cryopreservation using $\mathrm{CPT}^{\mathrm{TM}}$ vacutainer tubes containing a citrate anticoagulant that lies above a polyester barrier in the tube and a Ficoll-Hypaque density gradient lies below it. This tube allows for both sterile blood collection and density-based cell separation in a single container. Centrifugation of the $\mathrm{CPT}^{\mathrm{TM}}$ tube yielded a layer of PBMCs that was transferred under sterile conditions in preparation for cryopreservation. The final cell suspension was dispensed into aliquots in cryovials that were placed at $-80^{\circ} \mathrm{C}$ using controlled rate freezing to gradually lower the temperature of the suspension to $-80^{\circ} \mathrm{C}$ in preparation for final storage conditions in liquid nitrogen vapors at $-140^{\circ} \mathrm{C}$ to $-180^{\circ} \mathrm{C}$.

NOTE: Work in a biological safety cabinet. Use sterile technique throughout.

\section{Materials}

HBSS-PS (see recipe)

Hemolytic buffer (see recipe)

$20 \%$ FCS (see recipe)

Supplemented RPMI (HEPES) buffer (see recipe) 
$20 \%$ DMSO (see recipe)

Liquid nitrogen

Vacutainer $\mathrm{CPT}^{\mathrm{TM}}$ (cell preparation tube with sodium citrate, Becton Dickinson, cat. no. 362761)

Benchtop centrifuge

5-, 10-, and 25-ml disposable sterile pipets

50 -ml conical tubes, sterile

Hemacytometer (Bright Line, Improved Neubauer, 0.1-mm deep)

American Optical Zeiss microscope, bi-pin (low pressure), $10 \times$ ocular

Shallow trays

Cryovial holder

2-ml sterile cryovials, sterile, screw cap

Cardboard box

Freezing container (Mr. Frosty, Nalgene)

\section{Isolate PBMCs}

1. Keep $\mathrm{CPT}^{\mathrm{TM}}$ tubes at room temperature and in an upright position until they are ready to process for $30 \mathrm{~min}$ after unpacking.

During shipment, it is not always possible to control the tube temperature (i.e., high ambient temperature in summer or low ambient temperature in winter). It is crucial for the proper functionality of the CPT Ficoll layer that the processing be done at room temperature between $20^{\circ} \mathrm{C}$ and $25^{\circ} \mathrm{C}$. Incubating tubes 30 min at room temperature allows the tube temperature to equilibrate prior to processing.

2. Mix $\mathrm{CPT}^{\mathrm{TM}}$ tubes by inverting gently eight to ten times prior to centrifugation. Centrifuge $30 \mathrm{~min}$ at $1700 \times \mathrm{g}$, room temperature.

Centrifugation time is increased to 30 min compared to the 20 min recommended by the manufacturer to reduce contamination of the PBMC preparation by red blood cells.

3. Following centrifugation, ensure that proper separation of tube components by density gradient has occurred. From top to bottom, separation should show a plasma layer, PBMC layer, gel layer, Ficoll layer, and then red blood cells (Fig. 1).

4. Using a sterile 10-ml pipet, aspirate plasma on the gel by tilting the tube and having the pipet tip touch the side of the tube, not the gel barrier. Transfer all of plasma from the $\mathrm{CPT}^{\mathrm{TM}}$ tube into one labeled 50-ml conical tube.

5. Using a new, sterile 5-ml pipet, add $3 \mathrm{ml}$ HBSS-PS to each $\mathrm{CPT}^{\mathrm{TM}}$ tube by dispensing it down the side of the $\mathrm{CPT}^{\mathrm{TM}}$ tube to rinse tube, followed by gently pipetting up and down two times to ensure capture of all cells. Using the same pipet, avoiding pipetting gel with the liquid, transfer all HBSS-PS/sample mixture to the 50-ml conical tube containing original plasma.

6. Using a new, sterile 25-ml pipet, add additional HBSS-PS to bring volume in the conical tube to $35 \mathrm{ml}$.

7. Centrifuge conical tubes $10 \mathrm{~min}$ at $330 \times g$, room temperature. After centrifugation, visually check for an intact pellet. Pour off supernatant and disperse cell pellet by gently tapping tube or gently "racking" (racking involves drawing each conical tube at a $45^{\circ}$ angle across the top of a plastic tube rack).

Do not vortex samples to minimize damage to cells.

8. Using a sterile $5-\mathrm{ml}$ pipet, add $3 \mathrm{ml}$ hemolytic buffer and gently mix. Incubate $5 \mathrm{~min}$ at room temperature, then add $3 \mathrm{ml}$ HBSS-PS using a new 5-ml sterile pipet and invert or mix gently. 


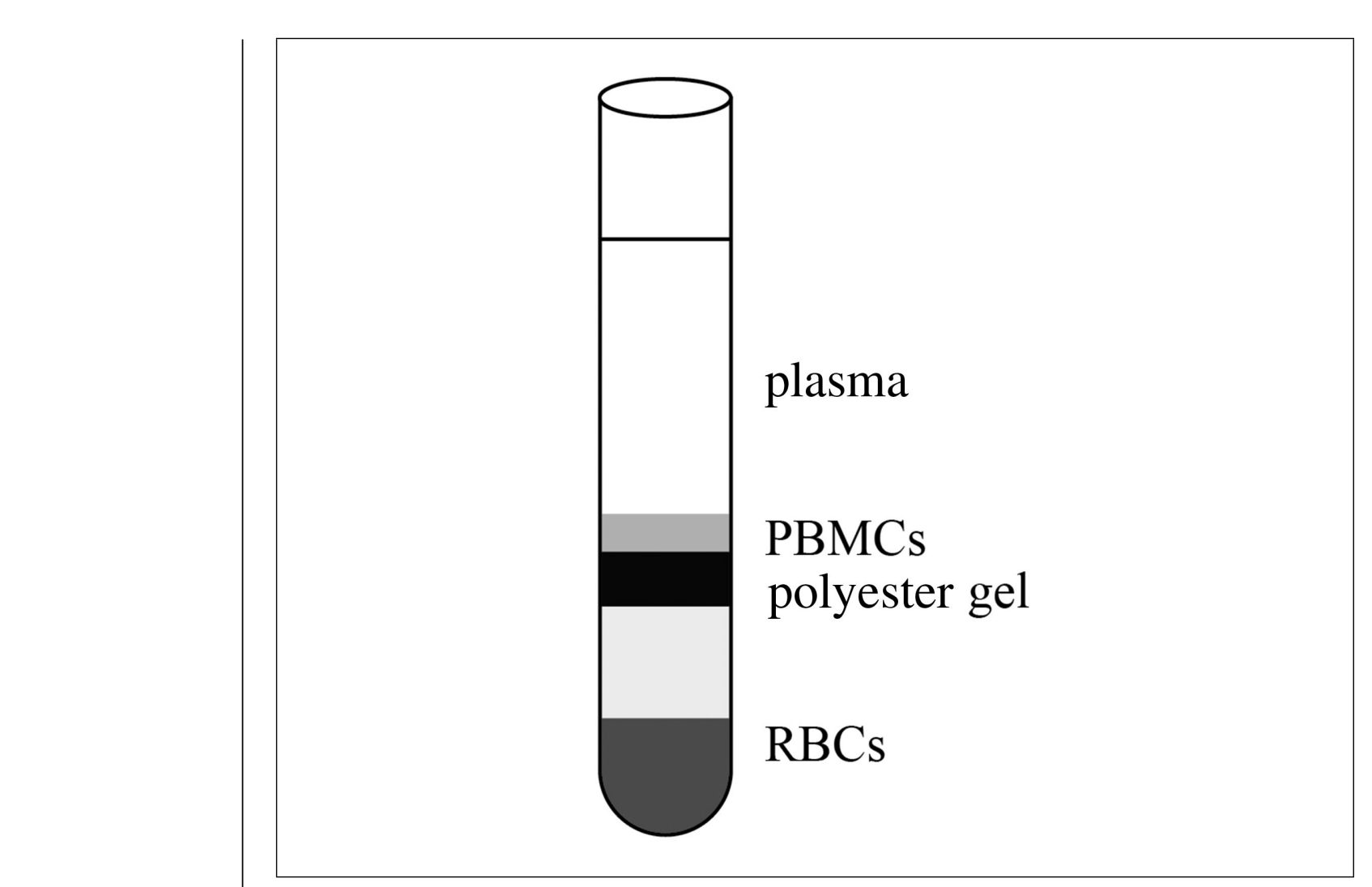

Figure 1 Separation of blood component using a CPT ${ }^{\mathrm{TM}}$ vacutainer. The diagram shows the separation of the blood component after centrifugation of the CPT ${ }^{\mathrm{TM}}$ tube. The plasma (white) lies on top of the PBMCs layer (light grey). Below the PBMC is the polyester gel (black). At the bottom of the tube, the red blood cells (RBC, dark grey) within the density gradient layer can be found.

When $C P T^{T M}$ tubes are used within the recommended time guidelines from the manufacturer, there is very little red blood cells contaminant within the PBMC layer; however, due to the time delay of 24 to $48 \mathrm{hr}$, contamination of the PBMC layer by RBC is more likely, therefore, an RBC lysis step was included.

9. Centrifuge $10 \mathrm{~min}$ at $330 \times \mathrm{g}$, room temperature. Visually check for an intact pellet to ensure that proper centrifugation has occurred. Pour off supernatant and disperse cell pellet by gently tapping tube or gently "racking" (see step 7; do not vortex).

10. Add $10 \mathrm{ml}$ HBSS-PS to cell pellet.

\section{Cryopreserve PBMCs}

11. Pipet $10 \mu \mathrm{l}$ of resuspended PBMCs from step 11 onto a hemacytometer.

12. Count cells located in the four outer squares of the hemacytometer using an optical microscope and calculate cell count using the following equation:

total cell number $=$ cell count $\times 16 \times$ resuspension volume $\times 2500$

13. Calculate the total volume of $20 \%$ DMSO needed for a final cell concentration of $4 \times 10^{6}$ cells $/ \mathrm{ml}$ using the following equation. Use this volume to resuspend the PBMCs in steps 16 and 18.

$$
\text { volume of medium }(\mathrm{ml})=\text { total cell number } /\left(4 \times 10^{6}\right)
$$

Cryopreservation and Immunophenotyping for Population Studies

14. Centrifuge cell suspension $10 \mathrm{~min}$ at $330 \times \mathrm{g}$, room temperature. 
15. Pour off supernatant. Gently disperse cell pellet. Add appropriate amount (half of the volume calculated in step 14) of $20 \%$ FCS in supplemented RPMI with HEPES.

16. For the freezing procedure, prepare a melting ice bath in a shallow tray. Place cryovial holder containing a labeled 2-ml cryovial into ice bath. Prepare a second ice bath. Place the 50-ml conical tube containing lymphocyte suspension into the second ice bath. Pipet the estimated amount of $20 \%$ DMSO in RPMI supplemented with HEPES (half of the volume calculated in step 14) into a labeled 50-ml conical tube and place this into the ice bath with the specimen.

17. Dilute cell suspension with an equal volume of cold 20\% DMSO (1:1 ratio of RPMI to DMSO). Using a sterile pipet, very slowly add the appropriate volume (calculated in step 14) dropwise with continuous mixing of the suspension tube to avoid concentration gradients of DMSO.

18. Using the same pipet, transfer $1 \mathrm{ml}$ of suspension into the appropriate pre-chilled, labeled 2-ml cryovial. Screw caps tightly. Keep cryovials on ice water until ready to freeze. Do not allow cells to sit in DMSO for $>30$ min prior to freezing.

19. Transfer vials into a cardboard box, keeping in upright position. Place box in a styrofoam container (controlled-rate freezer or a Mr. frosty container may be used if available). Place box at $-80^{\circ} \mathrm{C}$ and transfer cryovials to liquid nitrogen after a minimum of $8 \mathrm{hr}$ or maximum of $24 \mathrm{hr}$ at $-80^{\circ} \mathrm{C}$ for long-term storage.

Typically, two to three cryovials (each containing 4 to 6 million PBMCs) are obtained from a single CPT ${ }^{T M}$ processed using the conditions described in this protocol.

\section{THAWING CRYOPRESERVED PBMCS FOR IMMUNOPHENOTYPING}

The protocol below describes how cryopreserved PBMCs were thawed and rested in preparation for immunophenotyping.

\section{Materials}

Cryopreserved PBMC vial (see Basic Protocol 1)

Complete RPMI (see recipe), prewarmed

$100 \mathrm{U} / \mu 1$ DNase (Life Technology, cat. no. 18047019), store until manufacturer expiration date at $-20^{\circ} \mathrm{C}$

$1 \times$ PBS (see recipe)

$37^{\circ} \mathrm{C}$ water bath

Transfer pipets

15-ml conical polypropylene tubes

Centrifuge

$37^{\circ} \mathrm{C}, 5 \% \mathrm{CO}_{2}$ water-jacketed incubator (95\% humidity)

1. Place a cryopreserved PBMC vial into a $37^{\circ} \mathrm{C}$ water bath. Submerge cryovial halfway in bath and thaw for $60 \mathrm{sec}$.

PBMCs were frozen at 4 million cells in $1 \mathrm{ml}$ freezing medium. The authors found that 60 sec was the optimal time to thaw frozen PBMCs. Larger volumes may require a longer duration in the bath. The thawing step can be done in batches of ten samples. Thawing larger batches of cryovials increases failure rate during immunophenotyping.

2. Slowly add $1 \mathrm{ml}$ of warmed $\left(37^{\circ} \mathrm{C}\right)$ complete RPMI dropwise to thawed PBMCs against the side of the cryovial using a transfer pipet. 
3. Pour thawed PBMCs in $5 \mathrm{ml}$ complete RPMI into a $15-\mathrm{ml}$ conical tube that has been warmed to $37^{\circ} \mathrm{C}$ (no pipetting). Wipe down the tube so that no water from the water bath is transferred along with cells.

Excessive pipetting during the thawing process is disruptive to some immune cell subsets. At this step, pipetting can be avoided by pouring the cells rather than pipetting them.

4. Rinse cryovials one time with $2 \mathrm{ml}$ of warm complete RPMI, pour PBMCs into the conical tube containing cell mixture (no pipetting).

5. Incubate PBMCs 5 min in $37^{\circ} \mathrm{C}$ water bath.

Since multiple samples are being thawed at once for the study, this allows all the samples to rest shortly prior to the spin to remove DMSO.

6. Centrifuge PBMCs $10 \mathrm{~min}$ at $330 \times g$, room temperature.

7. Pour off supernatant (this will leave a small amount of complete RPMI behind that aids in pellet resuspension in step 8).

8. Add $1 \mathrm{ml}$ of prewarmed complete RPMI with $50 \mathrm{U} / \mathrm{ml}$ DNase. Mix by flicking or racking the tube (avoid pipetting up and down).

9. Incubate PBMCs for $1 \mathrm{hr}$ in a $37^{\circ} \mathrm{C}, 5 \% \mathrm{CO}_{2}$ water-jacketed incubator $(95 \%$ humidity). Leave cap of the conical tube slightly loose so that gas transfer can occur.

There is no clear consensus on the amount of time the cells should be rested after thawing and prior to immunophenotyping. Resting PBMCs $1 \mathrm{hr}$ at $37^{\circ} \mathrm{C}$ was necessary to provide immunophenotyping results in cryopreserved PBMCs comparable to whole-blood immunophenotyping in monocytes. More details on resting are provided in the Commentary.

BASIC PROTOCOL 3

Cryopreservation and Immunophenotyping for Population Studies

\section{QUALITY CONTROL AND IMMUNOPHENOTYPING OF PBMCS}

Since two flow cytometers are used and immunophenotyping is performed over 18 months, several quality control steps that monitor flow cytometer, antibodies, and reproducibility across technicians are described. The steps of the staining protocol are also described.

\section{Materials}

CS\&T research beads (setup beads, BD Biosciences, cat. no. 655050), store until manufacturer's expiration date at $4^{\circ} \mathrm{C}$, away from light

$1 \times$ PBS (see recipe)

Ultra Rainbow Beads (URBs, Spherotech, cat. no. URFP-30-2), store until manufacturer's expiration date at $4^{\circ} \mathrm{C}$, away from light

Antibody cocktail (see Tables 1 and 2)

Viability dye (see recipe)

LSRII (BD Biosciences) with a 488-nm blue laser (4 colors), 633-nm red laser (2 colors), 407-nm violet laser (4 colors), and 355-nm UV laser ( 3 colors)

FORTESSA X-20 (BD Biosciences) with a 488-nm blue laser (4 colors), 633-nm red laser ( 2 colors), 407-nm violet laser (4 colors), 355-nm UV laser ( 3 colors), and 561-nm yellow laser ( 2 colors)

FACSDiva software

Centrifuge

$15-\mathrm{ml}$ conical polypropylene tube

5-ml round-bottom, $12 \times 75-\mathrm{mm}$ polystyrene tube (Corning, cat. no. 352058) 
1. Turn on flow cytometer and lasers if necessary.

2. Refill sheath tank, empty waste tank, and prime two times.

3. Set flow speed to low and fine adjust knob to the middle position (five turns). Run water for $5 \mathrm{~min}$.

4. After 20 min of warm up, add one drop of CS\&T setup beads to $350 \mu 1$ of $1 \times$ PBS in a $5-\mathrm{mL}$ round-bottom polystyrene tube.

5. Open cytometer setup page in FACSDiva and run beads. Ensure that appropriate lot number is used and follow prompts from software.

6. If the CS\&T beads pass, start next quality control step. If the CS\&T beads do not pass, call for service.

If the CS\&T reports are monitored closely, it is possible to detect changes in laser alignments before CS\&T fails and call for service. Slight increases either in bright beads CVs or in $\triangle P M T V$ s or changes in laser delay are indicative of potential shifts in laser alignments. It is preferred to catch this drift early so a service visit can be scheduled at a convenient time rather than as an emergency service call.

7. Add one drop of URBs to $350 \mu 1$ of $1 \times$ PBS.

8. Set up voltages for each detector so that the beads fall within a gate of choice for each detector. Use these voltages as a base to save the application settings in FACSDiva, which will allow the software to adjust the voltages for each detector for minimal changes in PMTV.

9. Use the application settings for each subsequent URB that will be run on the flow cytometer. Establish a range for the median fluorescence intensity (MFI) for each detector (this range will be used to check subsequent URB).

10. Run the URB on low with the fine adjust knob set at the middle (five turns).

11. Record 30,000 events.

12. For the URB to pass quality control, the MFI for all detectors of the blue and red lasers must be within $3 \%$ of the average calculated at the beginning of the study, and within $5 \%$ of the average for each detectors for the violet and UV lasers (Kalina et al., 2012).

13. Thaw a compensation PBMC vial, which will be processed as a sample (see below).

Vials of cryopreserved PBMCs were set aside from a healthy volunteer to be used daily as a compensation sample. Daily compensation does improve data quality and will avoid adjustment of compensation post data acquisition.

14. After the 1-hr rest, resuspend the compensation sample in $2 \mathrm{ml}$ of $1 \times \mathrm{PBS}$.

15. Add $100 \mu \mathrm{l}$ of cells to 20 pre-labeled 5 -ml round-bottom $12 \times 75-\mathrm{mm}$ polystyrene tubes one per each individual antibody listed in Tables 1 and 2 and an unstained sample.

Information about antibodies and viability dyes (i.e., manufacturer and product number) is listed in Tables 1 and 2. Store until manufacturer's expiration date at $4{ }^{\circ} \mathrm{C}$, away from light.

In addition to the standard quality control procedures to ensure reliability of flow cytometers, draw ten CPT ${ }^{T M}$ tubes from four healthy volunteers before the start of the study. PBMCs were isolated and aliquots were frozen at a concentration of 2 million cells/cryovial following the procedure described in Basic Protocol 1. A control range 
Table 1 Antibody List and Volumes Per Sample for Panel 1 that Characterize T and B Cells

\begin{tabular}{|c|c|c|c|c|}
\hline Marker & Clone & Fluorochrome & Provider (cat. no. $)^{a}$ & $\begin{array}{l}\text { Volume per } \\
\text { sample }(\mu \mathrm{l})\end{array}$ \\
\hline $\begin{array}{l}\text { Brilliant stain } \\
\text { buffer }\end{array}$ & NA & NA & $\mathrm{BD}(659611)$ & 55 \\
\hline Viability dye & NA & FVS 570 (PE) & BD (564995) & 12 \\
\hline CD3 & UCHT1 & $\mathrm{APC}$ & $\mathrm{BD}(555335)$ & 4.4 \\
\hline HLA-DR & G46-6 & PE-CF594 & $\mathrm{BD}(562331)$ & 1.1 \\
\hline CD19 & $\mathrm{SJ} 25 \mathrm{C} 1$ & PE-Cy7 & $\mathrm{BD}(557835)$ & 1.1 \\
\hline CD27 & $\mathrm{O} 323$ & FITC & Biolegend (302806) & 2.75 \\
\hline CD8 & RPA-T8 & BUV395 & BD (563796) & 1.1 \\
\hline $\operatorname{IgD}$ & IA6-2 & BUV737 & $\mathrm{BD}(564687)$ & 1.1 \\
\hline CCR7 & G043H7 & BV421 & Biolegend (353208) & 2.75 \\
\hline $\mathrm{CD} 28$ & CD28.2 & BV510 & Biolegend (302936) & 2.75 \\
\hline CD95 & DX2 & BV605 & Biolegend (305628) & 2.75 \\
\hline CD45RA & HI100 & BV711 & Biolegend (304138) & 2.75 \\
\hline CD4 & RPA-T4 & APC-Cy7 & $\mathrm{BD}(557871)$ & 1.1 \\
\hline
\end{tabular}

${ }^{a} \mathrm{BD}, \mathrm{BD}$ Biosciences.

Table 2 Antibody List and Volumes Per Sample for Panel 2 that Characterize Dendritic Cells, NK, and Monocytes

\begin{tabular}{lllll}
\hline Marker & Clone & Fluorochrome & $\begin{array}{l}\text { Provider } \\
\text { (cat. no. })\end{array}$ & $\begin{array}{l}\text { Volume per } \\
\text { sample }(\mu 1)\end{array}$ \\
\hline Brilliant stain buffer & NA & NA & BD (659611) & 55 \\
Viability dye & NA & FVS 570 (PE) & BD (564995) & 12 \\
CD3 & UCHT1 & APC & BD (555335) & 4.4 \\
HLA-DR & G46-6 & PE-CF594 & BD (562331) & 1.1 \\
CD19 & SJ25C1 & PE-Cy7 & BD (557835) & 1.1 \\
CD11c & B-ly6 & BB515 & BD (564490) & 1.1 \\
CD20 & 2H7 & BUV395 & BD (563781) & 1.1 \\
CD16 & 3G8 & BUV737 & BD (564433) & 1.1 \\
CD56 & NCAM16.2 & BV421 & BD (562751) & 1.1 \\
CD14 & MOP9 & BV510 & BD (563079) & 1.1 \\
CD123 & 9F5 & BV711 & BD (563161) & 1.1 \\
CD45 & 2D1 & APC-Cy7 & BD (560178) & 0.55 \\
\hline
\end{tabular}

${ }^{a} \mathrm{BD}, \mathrm{BD}$ Biosciences.

Cryopreservation and Immunophenotyping for Population Studies

(mean \pm 2 standard deviations) was established before starting the study with four technicians processing and analyzing each of the four controls three times each (12 replicates/control). These controls were subsequently used to monitor antibody stocks, individual technique, and to train new flow technicians.

Prior to running study samples, new team members should process and analyze each control at least three times and be certified to analyze study samples only after all values are within the pre-established range. As experimental reproducibility controls, each experimenter should process one control per week along the HRS samples and rotate through the four controls in 1 month. The controls are immediately analyzed to 
ensure that all cell subsets are within the established ranges. If two cell populations in a particular control are outside established ranges, analyze study samples on that day, and have the same technician repeat the control on the following day. If the cell populations continue to be out of range, then no study samples are analyzed and data from study samples analyzed the previous day are discarded. If three populations are out of range, analyze study samples only after controls are repeated and pass established quality control parameters on the following day. If controls do not pass on 2 consecutive days, the flow cytometer should be serviced prior to study samples being analyzed.

\section{Stain for immunophenotyping}

16. Prepare antibody cocktail for panels 1 and 2 as described in Tables 1 and 2, respectively.

The antibody cocktail (antibody clone choices and fluorochrome choices) was optimized using a combination of the classic fluorescence minus one (FMO) experiments and a series of titration experiments. The cocktail can be prepared once a day and stored at $4^{\circ} \mathrm{C}$ in the dark. If this cocktail is going to be stored for longer than $4 \mathrm{hr}$, further validation is required.

a. Prepare a pre-mix with antibodies and brilliant stain buffer (required when two or more brilliant fluorochrome conjugated antibodies are used) for samples, prepare $10 \%$ overage (see Tables 1 and 2 for specific volumes).

b. Add viability dye to tubes of cells after the panel mix is added.

It is used slightly below the manufacturer's recommended amount.

17. At the end of the 1-hr PBMC rest (see Basic Protocol 2, step 9), add $10 \mathrm{ml}$ of $1 \times$ PBS (room temperature) to wash cells prior to staining.

18. Centrifuge cells $10 \mathrm{~min}$ at $330 \times g$, room temperature.

19. Pour off supernatant and pipet the remaining supernatant completely.

20. Resuspend cells in an appropriate volume of $1 \times$ PBS to achieve a final volume of $200 \mu$ l. Mix by flicking the tube.

Depending on the level of experience of the technician, the volume of $1 \times P B S$ added can vary slightly. The point is to minimize the volume of wasted cells and be as close to the final volume of $200 \mu \mathrm{l}$. The volume of $1 \times P B S$ required is generally between 160 and $180 \mu l$.

21. Add $100 \mu \mathrm{l}$ of cells to two pre-labeled 5-ml round-bottom polystyrene tubes (panel 1 and panel 2).

22. Add $71.5 \mu \mathrm{l}$ of panel 1 premix or $62.5 \mu \mathrm{l}$ of panel 2 premix to the appropriate tubes. Mix tube gently by agitating the tube rack.

It is crucial to ensure that all liquid (cells and antibody panel mix) is at the bottom of the tube. Any cells potentially stuck to the side of the tube will remain unlabeled and will be erroneously counted in the negatively stained fraction.

23. Add $12 \mu \mathrm{l}$ of viability dye to each tube.

24. Incubate exactly $20 \mathrm{~min}$ at room temperature protected from light.

25. Add $800 \mu \mathrm{l}$ of $1 \times$ PBS.

26. Centrifuge cells $10 \mathrm{~min}$ at $330 \times g$, room temperature.

27. Pipet off supernatant. Resuspend stained PBMCs in $500 \mu 1$ of $1 \times$ PBS and store on ice in the dark until flow cytometry. Run samples within $4 \mathrm{hr}$ of staining. 


\section{REAGENTS AND SOLUTIONS}

\section{Complete RPMI}

To prepare $500 \mathrm{ml}$ :

$445 \mathrm{ml} \mathrm{1 \times} \mathrm{RPMI} \mathrm{(Gibco,} \mathrm{cat.} \mathrm{no.} \mathrm{11875093)}$

$50 \mathrm{ml}$ fetal bovine serum (10\%) (FBS, Invitrogen, cat. no. 10437-028)

$5 \mathrm{ml}$ penicillin/streptomycin (100 U/ml) (PS, Gibco, cat. no. 15140-122)

Store 7 days at $4^{\circ} \mathrm{C}$

\section{DMSO, $20 \%$}

To the top of a $250-\mathrm{ml}$ filter flask, add $200 \mathrm{ml}$ of $20 \%$ FCS (see recipe). While slowly swirling a pipet in the FCS, add $50 \mathrm{ml}$ dimethyl sulfoxide (DMSO, Sigma, cat. no. D5879) to the FCS by using a disposable pipet (DMSO must be added slowly or protein will precipitate and the filter will clog). Store 7 days at $4^{\circ} \mathrm{C}$.

\section{FCS, $20 \%$}

To the top of a 500-ml sterile filter flask, add $400 \mathrm{ml}$ supplemented RPMI with HEPES, and then add $100 \mathrm{ml}$ fetal calf serum (FCS) (also called fetal bovine serum, FBS, Invitrogen, cat. no. 10437-028). Filter and store 7 days at $4{ }^{\circ} \mathrm{C}$.

\section{HBSS-PS}

$5 \mathrm{ml}$ penicillin/streptomycin (PS: 10,000 U/ml penicillin/10,000 $\mu \mathrm{g} / \mathrm{ml}$ streptomycin, Gibco, cat. no.15140-122)

$495 \mathrm{ml} 1 \times$ calcium and magnesium-free Hank's balanced salt solution (HBSS, Gibco, cat. no. 14170-112)

Store 30 days at $4^{\circ} \mathrm{C}$

\section{Hemolytic buffer}

$8.3 \mathrm{~g} \mathrm{NH}_{4} \mathrm{Cl}$ (500-g bottle, dry powder)

$1.0 \mathrm{~g} \mathrm{NaHCO}_{3}$ (500-g bottle, dry powder)

$0.04 \mathrm{~g}$ disodium EDTA (Fisher, cat. no. S311-100)

Dilute to $1000 \mathrm{ml}$ with sterile water

Sterilize by filtration in $0.45-\mu \mathrm{m}$ filter flask

Store 6 months at $4^{\circ} \mathrm{C}$

\section{PBS, $1 \times$}

Add 2 liters of $10 \times$ phosphate buffered saline, pH 7.4 (PBS, Lonza, cat. no. 17515Q) to 18 liters of deionized water. Store until manufacturer's expiration date at room temperature.

\section{Supplemented RPMI (HEPES) buffer}

$5 \mathrm{ml}$ heparin sodium injection, USP (Sagent, cat. no. NDC 25021-400-10)

$5 \mathrm{ml}$ penicillin/streptomycin (PS: 10,000 U/ml penicillin/10,000 $\mu \mathrm{g} / \mathrm{ml}$ streptomycin, Gibco, cat. no. 15140-122)

$5 \mathrm{ml}$ thawed $100 \times$ L-glutamine (mix well prior to addition; Gibco, cat. no. 25030-081)

Cryopreservation and Immunophenotyping for Population Studies $480 \mathrm{ml}$ 1× RPMI medium 1640 (RPMI with HEPES, Gibco, cat. no. 22400-89) Store 7 days at $4^{\circ} \mathrm{C}$ 
$594 \mu \mathrm{l} 1 \times$ PBS (see recipe)

$6 \mu$ viability dye stock (Viability Dye-FVS 450, BD Biosciences, cat. no. 564995), prepared according to manufacturer's recommendations

Store 90 days at $-20^{\circ} \mathrm{C}$

\section{COMMENTARY}

\section{Background Information}

Numerous publications have reported on the effect of cryopreservation on various immune subsets (Draxler, Madondo, Hanafi, Plebanski, \& Medcalf, 2017; Lemieux et al., 2017; Verschoor, Kohli, \& Balion, 2017). The two major types of cryopreservation evaluated across studies are cryopreserved whole blood and PBMCs isolated via a density gradient and then cryopreserved. Several studies compared the viability and functionality of cryopreserved whole blood and cryopreserved PBMCs. DNA repair capacity and mutagen sensitivity assays showed similar results in cryopreserved whole blood and cryopreserved PBMCs (Cheng, Wang, Spitz, \& Wei, 2001). While it is possible to stain immune cells for surface markers for immunophenotyping, fix, and then freeze at a lower temperature $\left(-20^{\circ} \mathrm{C}\right.$ or $\left.-80^{\circ} \mathrm{C}\right)$, this method does not result in viable cells for future functional assays (Pinto et al., 2016; Madelaine Paredes, Tadaki, Sooter, Gamboni, \& Sheppard, 2017). Studies have also evaluated different methods for thawing samples with some studies thawing samples and performing immunophenotyping without any rest period, while other studies had rest periods (incubating cells at $37^{\circ} \mathrm{C}$ ) of 1 to $24 \mathrm{hr}$ prior to immunophenotyping (Lemieux et al., 2017; Wang et al., 2016, Kutscher et al., 2013). In general, these studies have shown that a short rest period of $1 \mathrm{hr}$ generally improves comparability of various immune subsets in cryopreserved cells and fresh cells (Lemieux et al., 2017), although a longer rest period of up to $24 \mathrm{hr}$ results in higher proportion of activated cells in the cryopreserved sample (Sattui et al., 2012). An 18-hr rest period reduced the number of apoptotic cells in thawed PBMCs and positively affected lymphocyte functionality (Wang et al., 2016). Published effects of cryopreservation and thawing procedures on the enumeration and functions of individual immune subsets are reviewed below.

\section{T cells}

Since $\mathrm{T}$ cells and their subsets are useful in a number of applications such as monitoring HIV patients (Sattui et al., 2012; Zhang,
Nilles, Johnson, \& Margolick, 2016), functional studies (Cheng et al., 2001), activation studies (Lemieux et al., 2017), and vaccine testing (Kutscher et al., 2013; Wang et al., 2016), the effect of cryopreservation on $T$ cells has been extensively investigated. $\mathrm{Nu}-$ merous studies showed no effect of cryopreservation in the enumeration of $\mathrm{T}$ cells, helper and cytotoxic T cells (Draxler et al., 2017; Weinberg et al., 2010; Verschoor et al., 2017; Sattui et al., 2012). Though cryopreservation did not affect the distribution of helper and cytotoxic T cells, some $\mathrm{T}$ cell subsets such as 'naive helper,' 'central memory helper,' and 'cytotoxic' were significantly lower and cytotoxic effector $\mathrm{T}$ cells were higher in cryopreserved samples compared to fresh whole blood (Costantini et al., 2003). A significant decrease in $\mathrm{T}$ cells and helper $\mathrm{T}$ cells observed after cryopreservation was not rescued by a 1-hr rest (Lemieux et al., 2017). Although there were significant differences in several $T$ cell subsets when immunophenotyping was performed on cryopreserved PBMCs without resting the cells, the proportion of naïve, central memory, effector, effector memory, Th1, and Th2 as well as activated subsets within the helper and cytotoxic $T$ cell populations did not show any significant change with cryopreservation after a rest period (Lemieux et al., 2017). In Lemieux's study, a 1-hr rest period also showed greater concordance of helper and cytotoxic $\mathrm{T}$ cell subsets with the fresh sample than with the sample that was rested for $24 \mathrm{hr}$ or not rested at all (Lemieux et al., 2017). In contrast, a rest period of $18 \mathrm{hr}$ after thawing improved lymphocyte functionality. Smaller T cell subsets such as regulatory helper $\mathrm{T}$ cells (Tregs) and naïve helper or naïve cytotoxic $\mathrm{T}$ cells measured levels are comparable between cryopreserved PBMCs and fresh blood (Wang et al., 2016). Tregs play a major role in immune homeostasis and down-regulate both Th1 and Th2 responses (Zhang, Zhang, Gu, $\mathrm{He}$, \& Sun, 2014) and are sensitive to cryopreservation. Though helper $\mathrm{T}$ cell enumeration remained constant between fresh and frozen PBMCs, Treg counts were reduced in cryopreserved PBMCs regardless of HIV status (Sattui et al., 2012). However, in this study, the
Phenotypic Analysis 11 of 16

Supplement 84 
PBMCs were not rested after being thawed. Lemieux (2017) showed that a rest of $1 \mathrm{hr}$ was sufficient to restore $\mathrm{T}$ cell phenotype in thawed PBMCs, making it comparable to fresh samples. They also showed that using a more sensitive flow data analysis method (viSNE) compared the more conventional flow analysis method (FlowJo or FCS express) could improve detection of poorly represented populations such as Tregs (Lemieux et al., 2017). Resting thawed PBMCs for $4 \mathrm{hr}$ has also been shown to restore CD120b expression on Tregs regardless of HIV status (Zhang et al., 2016), although a number of Tregs did not seem to be affected by either cryopreservation or duration of the rest period after thawing. Other studies showed that Tregs were decreased, but highly correlated, in cryopreserved samples (Elkord, 2009) without any resting of thawed samples.

\section{B cells}

B cells can be successfully isolated and immortalized in thawed whole blood that was cryopreserved even after a delay of $24 \mathrm{hr}$ after blood collection (Peakman and Elliot, 2008). $\mathrm{B}$ cells are increased in cryopreserved blood compared to a fresh sample (Verschoor et al., 2017). The increase in B cells in cryopreserved PBMCs observed by Lemieux et al. remained unchanged by either a 1- or 24-hr rest period (Lemieux et al., 2017).

\section{NK cells}

NK cell frequencies are lower in cryopreserved PBMCs but still highly correlated in cryopreserved blood compared to fresh blood (Verschoor et al., 2017). A rest period after thawing does not rescue the NK cell enumeration in cryopreserved PBMCs (Lemieux et al., 2017). In contrast, another study that included no rest period after thawing found that NK cell enumeration was not affected by cryopreservation of PBMCs compared to fresh blood (Draxler et al., 2017). The reasons for the discrepancy between these studies are unclear.

\section{Monocytes}

Two studies showed that monocyte counts were more elevated in cryopreserved blood or cryopreserved PBMCs compared to fresh blood (Draxler et al., 2017; Verschoor et al., 2017). However, neither one of these studies allowed the cells to rest prior to staining. The proportion of monocytes in cryopreserved PBMCs was similar to fresh blood after both 1 and $24 \mathrm{hr}$ of rest after thawing (Lemieux et al., 2017).

\section{Dendritic cells}

Dendritic cells are some of the least represented cells in PBMCs and are sensitive to freeze/thaw cycles. Makino and Baba (1997) showed that upon proper cryopreservation conditions (25\% FBS and 10\% DMSO), dendritic cells could be frozen and properly thawed and cultured in the same manner as for freshly isolated PBMCs. But while the dendritic cells retain their function after cryopreservation, they appear to increase in numbers (Draxler et al., 2017; Verschoor et al., 2017). Dendritic cell subsets are affected by PBMC manipulation. A study showed that Ficoll separation, regardless of cryopreservation status, increases the ratio of plasmacytoid dendritic to myeloid dendritic cells (Gerrits et al., 2007). These cells also had a higher expression level of maturation markers.

\section{Critical Parameters and Troubleshooting}

\section{Standardization of cryopreservation and thawing procedures}

To obtain reproducible results during immunophenotyping, it is important to minimize pre-analytical variability by standardizing cryopreservation and thawing of PBMCs. These include the following.

1. The time between blood collection and sample processing should remain consistently within $48 \mathrm{hr}$ as viability of cells after cryopreservation is dramatically reduced if the delay between sample collection and processing is $>48 \mathrm{hr}$ (data not shown).

2. Although $\mathrm{CPT}^{\mathrm{TM}}$ tubes are shipped in styrofoam containers with room temperature gel packs, it is critical to keep the tubes upright at room temperature for $30 \mathrm{~min}$ prior to processing so that the gel layer can equilibrate to room temperature.

3. Rate of cooling during sample freezing is an important step. The cooling rate must be maintained at its optimum rate as cell survival will decrease if more or less of the optimal cooling rate is used (Hubel \& Skubitz, 2017). The PBMC cryovials were stored in a styrofoam container at $-80^{\circ} \mathrm{C}$ to control the cooling rate. One can also use a freezer specifically designed to cryopreserve cells or a Mr. Frosty (Nalgene), which is a container using the cooling property of isopropanol to maintain an optimum cooling rate of $1^{\circ} \mathrm{C} / \mathrm{min}$.

4. Using cold solutions during the cryopreservation process has been shown to improve viability and functional recovery of PBMCs (Tree, Roep, \& Peakman, 2004). When 
comparing the use of cold freezing medium versus room temperature freezing medium, Tree (2004) noted that PBMCs frozen using cold medium performed more similarly to fresh blood in a functional assay (e.g., T cell response to tetanus). Cold 20\% DMSO (in $1 \times$ RPMI) solution and cold $20 \%$ FBS (in $1 \times$ RPMI) were used to process the PBMCs.

5. Storage temperature, and more specifically changes in storage temperature, can affect cell viability and subsets recovery (Cosentino et al., 2003). Storing the control PBMCs and study samples in the same liquid nitrogen freezer allows evaluation of any differences in subset recovery or viability due to changes in storage temperature.

6. Fast thawing at $37^{\circ} \mathrm{C}$ is an important step to standardize across technicians. A thawing time of $60 \mathrm{sec}$ to maintain consistency between technicians is recommended.

7. Based on previously published data (Lemieux et al., 2017), cells were rested $1 \mathrm{hr}$ at $37^{\circ} \mathrm{C}$ after thawing to restore most immune cell subsets to their level in fresh blood. The cell subsets of interest are the major determinant of whether to include a rest period and to decide on the duration of the rest period.

8. Appropriate training of technicians is key to standardization. While $\mathrm{CPT}^{\mathrm{TM}}$ vacutainers are easier to use than a Ficoll gradient, cryopreserving PBMCs still requires a certain level of technical skill. The technicians processing the samples for cryopreservation were trained by a senior member of the staff on all aspects of the procedure (cell count, cooling of samples, recording of reagent lot numbers, etc.). A senior staff member then observed trainees closely before being allowed to work on study samples. A similar training method was used in the flow laboratory. To monitor proper thawing technique, HRS controls were used as a test for appropriate and timely technique.

\section{Standardization of immunophenotyping procedures}

Variability in immunophenotyping assays can be minimized using the following procedures.

1. Standardize flow cytometer configurations across multiple instruments. For example, both flow cytometers used for HRS were equipped with similar lasers (blue, UV, red, and violet) and the same filters for each PMT.

2. Establish a daily thorough flow cytometer quality control protocol as described in $\mathrm{Ba}$ sic Protocol 3. Minimize downtime by carefully monitoring CST and URB to catch laser drift prior to outright quality control failure. Use frozen PBMCs from a healthy volunteer to perform daily compensation setup and calculations.

3. During the experimental setup, perform all steps required to check the antibodies used in the study. This includes testing all antibodies in a dilution series and including all the FMO (fluorescence minus one) controls. If possible, purchase antibodies in bulk so that they are from the same lot. However, if that is not possible, test every new lot of reagents using control samples and ensure similar values for all subsets before using new lots of reagents on study samples. Use PBMC controls to check the consistency of panels over the course of the study.

4. In the context of immunophenotyping, use controls to check both reagents (antibodies and buffers) as well as technical abilities and consistency during the study. Train new technicians during the study. The PBMC controls proved to be an invaluable tool to test the readiness of each new technician. As described in Basic Protocol 3, have each new trainee immunophenotype each control three times on different days. If all measured immune populations were within the established range, the new trainee should start running HRS samples. If immune populations were out of range, additional training should be provided.

5. When running samples for HRS, group ten samples per batch for processing from thawing to immunophenotyping. Once all technicians become proficient, slowly increase the number of samples per batch (up to 16); however, an increase in the number of samples with low cell count (data not shown) has been observed. This increase in number of samples per batch only affected total thawing time. It is suspected that the increased time at $37^{\circ} \mathrm{C}$ before centrifuging and removing DMSO affected cell viability.

6. During initial panel testing, identify lower numbers of dendritic cells if the samples are kept on ice water rather than "regular ice." Process samples daily in five scaled batches of ten samples each to minimize sample time on ice (generally $2 \mathrm{hr}$ maximum per batch), which minimized variability in dendritic cell measurement.

7. The fluidics in the flow cytometer must be cleaned daily and a full clean of the fluidics should be performed monthly.

\section{Anticipated Results}

Using the cryopreservation procedures described in this unit, the central laboratory was
Phenotypic Analysis

\section{3 of 16}


able to process up to 80 samples daily and all samples were processed on the same day samples were received at the laboratory. Immunophenotyping of up to 50 samples daily following the abovementioned protocols was also achieved. The total failure rate was $\leq 5 \%$ when using the quality control procedures outlined in this unit.

\section{Time Considerations}

\section{Cryopreservation}

Cryopreservation of PBMCs is time consuming and requires an experienced technician. $\mathrm{CPT}^{\mathrm{TM}}$ vacutainers were chosen to save time over a Ficoll separation. It has been shown that viability and $\mathrm{T}$ cell subset enumeration is similar in cryopreserved PBMCs that have been isolated using either a $\mathrm{CPT}^{\mathrm{TM}}$ vacutainer or the Ficoll separation method (Ruitenberg, Mulder, Maino, Landay, \& Ghanekar, 2006). The protocol for cryopreservation of PBMCs (see Basic Protocol 1) is straightforward and can be done in $\sim 3 \mathrm{hr}$ for one $\mathrm{CPT}^{\mathrm{TM}}$ tube. Since the central laboratory received more than one sample a day, the separation and cryopreservation of PBMCs were batched. This only marginally increased processing time to $4 \mathrm{hr}$ per batch. If several trained technicians are assigned to cryopreservation and processing was staggered, upwards of 80 samples could be cryopreserved in 1 day without the need for automation.

\section{Flow cytometry set up}

To limit sample loss due to instrument failure, no samples are thawed until both (or at least one) flow cytometers have passed quality control procedures, which takes $\sim 40 \mathrm{~min}$. The lasers need to warm up for a minimum of $20 \mathrm{~min}$ prior to use. This allows enough time to refill both sheath tanks, empty waste tank, and prime the fluidic systems of the flows. After the CST has been run, set up the URB experiment and its associated application settings. The cryopreserved PBMCs used for compensation setup are also thawed during this time.

\section{PBMC preparation from thawing to immunophenotyping}

The study samples were processed in batches of ten samples from thawing to staining. During an 8-hr workday, two technicians can prepare five sets of ten samples without requiring the need for any automation and without compromising data quality. Immunophenotyping of PBMCs from removal of cryovials from liquid nitrogen to staining took $\sim 2 \mathrm{hr}$ with 35 to $40 \mathrm{~min}$ of hands-on work at the bench. By staggering the batches appropriately, minimal time has been achieved where the samples sit on ice and all 50 samples were processed during an 8-hr workday.

\section{Summary}

Previous studies indicate that cryopreserving whole blood or isolated PBMCs both yield similar results for immunophenotyping and functional studies. The effect of cryopreservation on various cell subsets has been described previously and use of a rest period after thawing can help restore immune cell distributions to those seen in fresh blood. Standardization of cryopreservation, thawing and immunophenotyping procedures and use of control samples, stored and analyzed in a manner identical to study samples, can significantly reduce variability of immunophenotyping measurements in large scale population studies.

\section{Acknowledgements}

This work was funded by a grant from the National Institute of Aging (U01 AG009740). The authors would like to thank Sharon Minnerath, Vicky Makky, and Eileen Studt for assistance with cryopreservation and Zach Flaten, Devon Hunter-Schlichting, Erin Cassidy, Paige Hartman, Tori Contreras, and Ramya Ramasubramian for assistance with immunophenotyping of cryopreserved samples.

\section{Conflict of Interest}

The authors have no conflict of interest to report.

\section{Literature Cited}

Cheng, L., Wang, L. E., Spitz, M. R., \& Wei, Q. (2001). Cryopreserving whole blood for functional assays using viable lymphocytes in molecular epidemiology studies. Cancer Letters, 166(2), 155-163. doi: 10.1016/ S0304-3835(01)00400-1.

Cosentino, L. M., Corwin, W., Baust, J. M., DiazMayoral, N., Cooley, H., Shao, W., Van Buskirk, R., \& Baust, J. G. (2007). Preliminary report: Evaluation of storage conditions and cryococktails during peripheral blood mononuclear cell cryopreservation. Cell Preservation Technology, 5(4). doi: 10.1089/cpt.2007.9987.

Costantini, A., Mancini, S., Giuliodoro, S., Butini, L., Regnery, C. M., Silvestri, G., \& Montroni, M. (2003). Effects of cryopreservation on lymphocyte immunophenotype and function. Journal of Immunological Methods, 278(1-2), 145-155. doi: 10.1016/S0022-1759(03)00202-3.

Draxler, D. F., Madondo, M. T., Hanafi, G., Plebanski, M., \& Medcalf, R. L. (2017). A flow cytometric analysis to efficiently quantify multiple innate immune cells and $\mathrm{T}$ cell subsets in human 
blood. Cytometry Part A, 91(4), 336-350. doi: 10.1002/cyto.a.23080.

Elkord, E. (2009). Frequency of human T regulatory cells in peripheral blood is significantly reduced by cryopreservation. Journal of Immunological Methods, 347(1-2), 87-90. doi: 10.1016/j.jim.2009.06.001.

Elliott, P., Peakman, T. C., \& UK Biobank (2008). The UK Biobank sample handling and storage protocol for the collection, processing and archiving of human blood and urine. International Journal of Epidemiology, 37(2), 234-244. doi: 10.1093/ije/dym276.

Friedman, G. D., Cutter, G. R., Donahue, R. P., Hughes, G. H., Hulley, S. B., Jacobs, D. R. Jr, ... Savage, P. J. (1988). CARDIA: Study design, recruitment, and some characteristics of the examined subjects. Journal of Clinical Epidemiology, 41(11), 1105-1116. doi: 10.1016/0895-4356(88)90080-7.

Gerrits, J. H., Athanassopoulos, P., Vaessen, L. M., Klepper, M., Weimar, W., \& van Besouw, N. M. (2007). Peripheral blood manipulation significantly affects the result of dendritic cell monitoring. Transplant Immunology, 17(3), 169-177. doi: 10.1016/j.trim.2006.11.006.

Hubel, A. \& Skubitz, A. P. N. (2017) Principles of Cryopreservation. In Biobanking of Human Biospecimens. (P. Hainaut, J. Vaught, K. Zatloukal, \& M. Pasterk, eds.), Springer, Cham, Switzerland.

Hughes, G. H., Cutter, G., Donahue, R., Friedman, G. D., Hulley, S., Hunkeler, E., Jacobs, D. R. Jr et al. (1987). Recruitment in the coronary artery disease risk development in young adults (Cardia) study. Controlled Clinical Trials, 8(4), 68S-73S. doi: 10.1016/0197-2456(87) 90008-0.

Kalina, T., Flores-Montero, J., van der Velden, V. H. J., Martin-Ayuso, M., Böttcher, S., Ritgen, M., \& Orfao, A. (2012). On behalf of the EuroFlow Consortium (EU-FP6, LSHB-CT-2006018708) EuroFlow standardization of flow cytometer instrument settings and immunophenotyping protocols. Leukemia, 26(9), 1986-2010. doi: 10.1038/leu.2012.122.

Kutscher, S., Dembek, C. J., Deckert, S., Russo, C., Körber, N., Bogner, J. R., . . B Bauer, T. (2013). Overnight resting of PBMC changes functional signatures of antigen specific $\mathrm{T}$ - cell responses: Impact for immune monitoring within clinical trials. PLoS One, 8(10) doi: 10.1371/journal.pone.0076215.

Landgren, O., Albitar, M., Ma, W., Abbasi, F., Hayes, R. B., Ghia, P., ... Caporaso, N. E. (2009). B-cell clones as early markers for chronic lymphocytic leukemia. New England Journal of Medicine, 360(7), 659-667. doi: 10.1056/NEJMoa0806122.

Lemieux, J., Jobin, C., Simard, C., \& Néron, S. (2017). A global look into human $T$ cell subsets before and after cryopreservation using multiparametric flow cytometry and two-dimensional visualization analysis. Journal of Immunological Methods, 434, 73-82. doi: 10.1016/j.jim.2016.04.010.

Makino, M., \& Baba, M. (1997). A cryopreservation method of human peripheral blood mononuclear cells for efficient production of dendritic cells. Scandinavian Journal of Immunology, 45(6), 618-622. doi: 10.1046/ j.1365-3083.1997.d01-441.x.

Madelaine Paredes, R., Tadaki, D. K., Sooter, A., Gamboni, F., \& Sheppard, C. D. R.F. (2017). Cryopreservation of human whole blood allows immunophenotyping by flow cytometry up to 30 days after cell isolation. Journal of Immunological Methods, 452, 32-38.

Peakman, T. C. \& Elliott, P. (2008). The UK Biobank sample handling and storage validation studies. International Journal of Epidemiology, 37(1), i2-6. doi: 10.1093/ije/dyn019.

Pinto, L. A., Trivett, M. T., Wallace, D., Higgins, J., Baseler, M., Terabe, M., ... Hildesheim, A. (2016). Fixation and cryopreservation of whole blood and isolated mononuclear cells: Influence of different procedures on lymphocyte subset analysis by flow cytometry. Journal of Immunological Methods, 434, 73-82.

Ruitenberg, J. J., Mulder, C. B., Maino, V. C., Landay, A. L., \& Ghanekar, S. A. (2006). Vacutainer CPT and Ficoll density gradient separation perform equivalently in maintaining the quality and function of PBMC from HIV seropositive blood samples. BMC Immunology, 7, 11. doi: 10.1186/1471-2172-7-11.

Sattui, S., de la Flor, C., Sanchez, C., Lewis, D., Lopez, G., Rizo-Patrón, E., ... Montes, M. (2012). Cryopreservation modulates the detection of regulatory $\mathrm{T}$ cell markers. Cytometry Part B Clinical Cytometry, 82(1), 54-58. doi: 10.1002/cyto.b.20621.

Stevens, V. L., Patel, A. V., Feigelson, H. S., Rodriguez, C., Thun, M. J., \& Calle, E. E. (2007). Cryopreservation of whole blood samples collected in the field for a large epidemiologic study. Cancer Epidemiology, Biomarkers \& Prevention, 16(10), 2160-2163. doi: 10.1158/1055-9965.EPI-070604.

Tree, T. I., Roep, B. O., \& Peakman, M. (2004). Enhancing the sensitivity of assays to detect $T$ cell reactivity: The effect of cell separation and cryopreservation media. Annals of the New York Academy of Sciences, 1037, 26-32. doi: 10.1196/annals.1337.005.

Verschoor, C. P., Kohli, V., \& Balion, C. (2017). A comprehensive assessment of immunophenotyping performed in cryopreserved peripheral whole blood. Cytometry Part B Clinical Cytometry, Apr 5 doi: 10.1002/cyto.b.21526.

Wang, L., Hückelhoven, A., Hong, J., Jin, N., Mani, J., Chen, B. A., .. Schmitt, A. (2016). Standardization of cryopreserved peripheral blood mononuclear cells through a resting process for clinical immunomonitoring-Development of an algorithm. Cytometry Part A, 89(3), 246258. doi: 10.1002/cyto.a.22813.
Phenotypic Analysis 15 of 16

Supplement 84 
Weinberg, A., Song, L. Y., Wilkening, C. L., Fenton, T., Hural, J., Louzao, R., ... Tustin, N. B. (2010). Optimization of storage and shipment of cryopreserved peripheral blood mononuclear cells from HIV-infected and uninfected individuals for ELISPOT assays. Journal of Immunological Methods, 363(1), 42-50. doi: 10.1016/j.jim.2010.09.032.

Zhang, W., Nilles, T. L., Johnson, J. R., \& Margolick, J. B. (2016). The effect of cellular isolation and cryopreservation on the expression of markers identifying subsets of regulatory T cells. Journal of Immunological Methods, 431, 31-37. doi: 10.1016/j.jim.2016.02 .004 .

Zhang, Y., Zhang, Y., Gu, W., He, L., \& Sun, B. (2014). Th1/Th2 cell's function in immune system. Advances in Experimental Medicine and Biology, 841, 45-65. doi: 10.1007/ 978-94-017-9487-9_3.

Cryopreservation and Immunophenotyping for Population Studies 